\title{
Identification and functional characterisation of zinc transporters in the human fungal pathogen Candida parapsilosis
}

Tibor Mihaly Nemeth ${ }^{1}$, Tamas Takacs ${ }^{1}$, Duncan Wilson ${ }^{2}$ and Attila Gacser ${ }^{1}$

1: University of Szeged, Szeged, Hungary

2: Medical Research Council Centre for Medical Mycology at the University of Aberdeen, Aberdeen Fungal Group, Aberdeen, United Kingdom

INTRODUCTION Candida parapsilosis is a diploid fungus belonging to CUG-clade of Ascomycota. It is a member of the normal human microbiota but can cause mild or severe infections particularly amongst low birthweight neonates under specific circumstances (1). During host - pathogen interaction microbes need to acquire not only carbon and nitrogen sources, but essential minerals as well. Indeed fighting for essential ions is so important that the host employs energy demanding processes to keep the concentration of free minerals as low as possible. This is known and characterised as nutritional immunity (2). Successfull pathogens have evolved specific mechanisms by which they become capable of acquiring growth limiting minerals upon interaction with the host. One of these trace elements is zinc, that plays a very important role as a cofactor of proteins (3). The way of zinc acquisition is a described process in Candida albicans, where a secreted zinc-binding protein Pra1 uptakes zinc ions from host zinc-chelator/binding proteins and delivers them to the fungal high affinity plasma membrane importer Zrt1 (4). Pra1 ortholog is not present in $C$. parapsilosis that suggests that this pathogen utilises a different mechanism for zinc acquisition during the interaction.

\begin{tabular}{|c|c|c|}
\hline $\begin{array}{l}\text { Zinc transporters in } \\
\text { Candida albicans }\end{array}$ & $\begin{array}{l}\text { Function of orthologs in } \\
\text { S. cerevisiae }\end{array}$ & $\begin{array}{c}\text { Orthologs in } C . \\
\text { parapsilosis }\end{array}$ \\
\hline CaZRT1 & $\begin{array}{l}\text { High affinity zinc ion } \\
\text { transporter }\end{array}$ & CPAR2_500170 \\
\hline \multirow[t]{3}{*}{ CaZRT2 } & $\begin{array}{l}\text { Low affinity zinc ion } \\
\text { transporter }\end{array}$ & $\begin{array}{c}\text { CpZRT2 } \\
\text { (CPAR2_210740) }\end{array}$ \\
\hline & (Paralog) & CPAR2_806710 \\
\hline & (Paralog) & CPAR2_806720 \\
\hline CaZRT3 & Vacuolar zinc exporter & CPAR2_212080 \\
\hline orf19.1536 (ZRC1) & Vacuolar zinc importer & $\begin{array}{c}\text { CpZRC1 } \\
\text { (CPAR2_212100) }\end{array}$ \\
\hline
\end{tabular}

To reveal the key components of the zinc uptake in C. parapsilosis an in silico analysis was performed to identify potential zinc transporters.

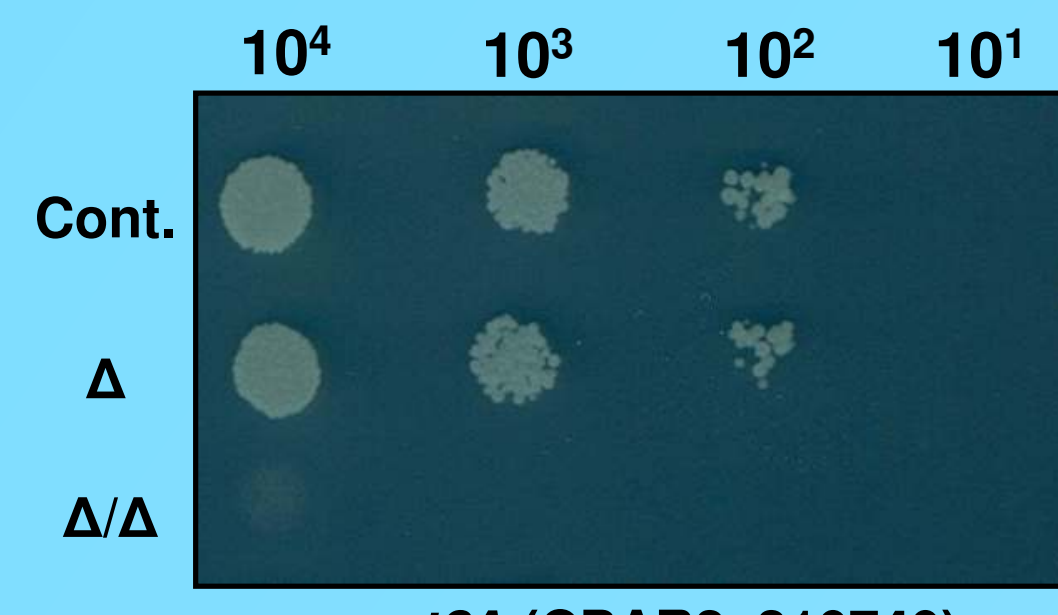

cpzrt2 $\triangle$ (CPAR2 210740)

$0.05 \mathrm{mM} \mathrm{ZnSO} 4,37^{\circ}, 2$ days

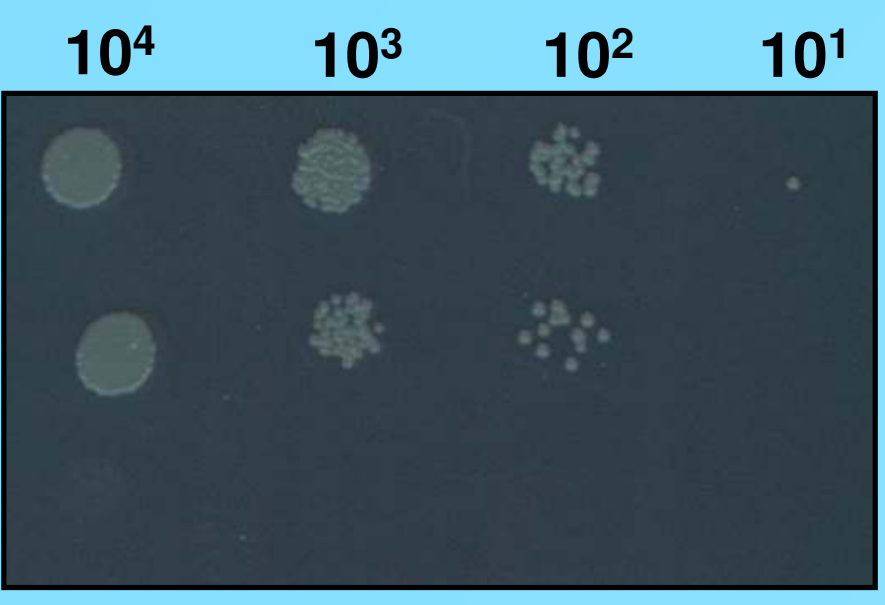

cpzrc1 1(CPAR2_212100)

$5 \mathrm{mM} \mathrm{ZnSO} 4,37^{\circ}, 2$ days
Knock-out mutants were generated and sentenced to phenotypic analyisis under different stress conditions as well as various zinc ion concentrations (5). The viability of only two out of six mutants differed from the control. At lower zinc ion concentration cpzrt2 $\Delta$ mutant while at higher concentration cpzrc1 1 strain showed growth deficiency.

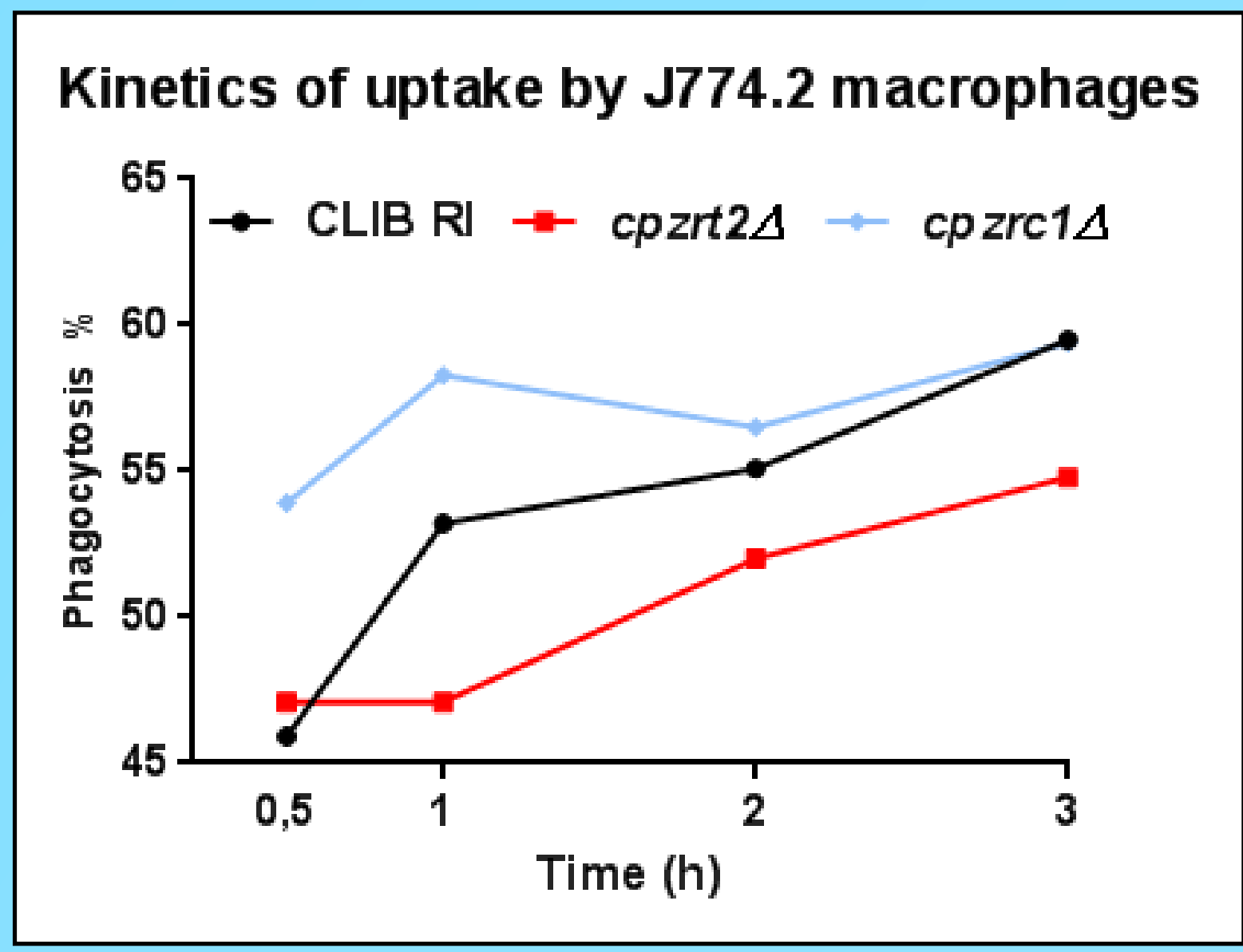

Mutant strains were incubated with $\mathbf{J 7 7 4 . 2}$ murine macrophages to record uptake kinetics with Amnis $₫$ FlowSight $₫$ flow cytometer. The cpzrt2 $\Delta$ mutant was more, while cpzrc1 $1 \Delta$ was less resistant to phagocytosis compared to the control in our model.

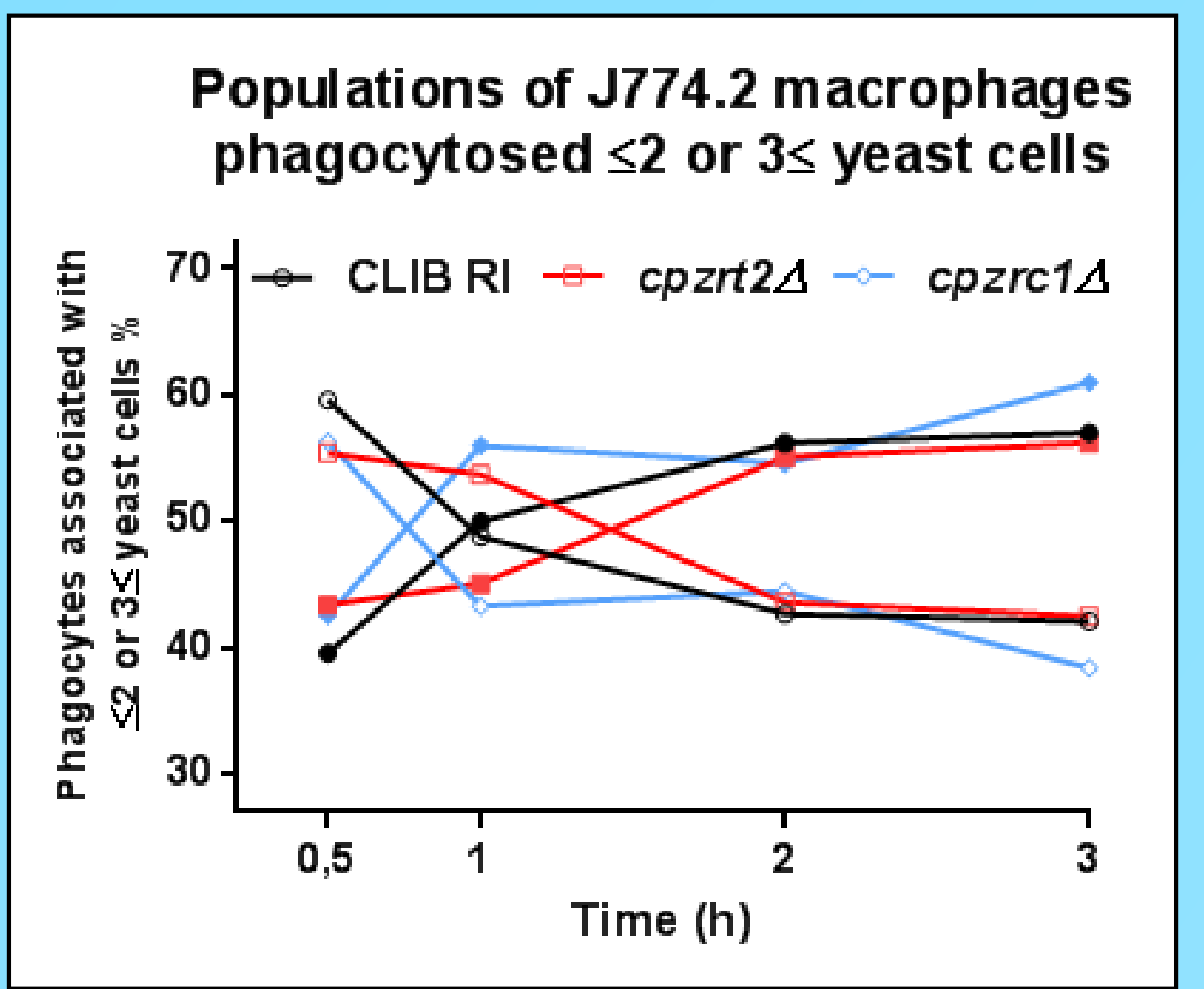

We also analysed the percentage of phagocytes associated with one or two (empty symbols) and more than two (solid symbols) fungal cells. The population of macrophages associated with at least three yeast cells formed more rapidly in the presence of cpzrc1 $1 \Delta$ - and slower upon challenging with cpzrt2 $\Delta$ mutants compared to the control.

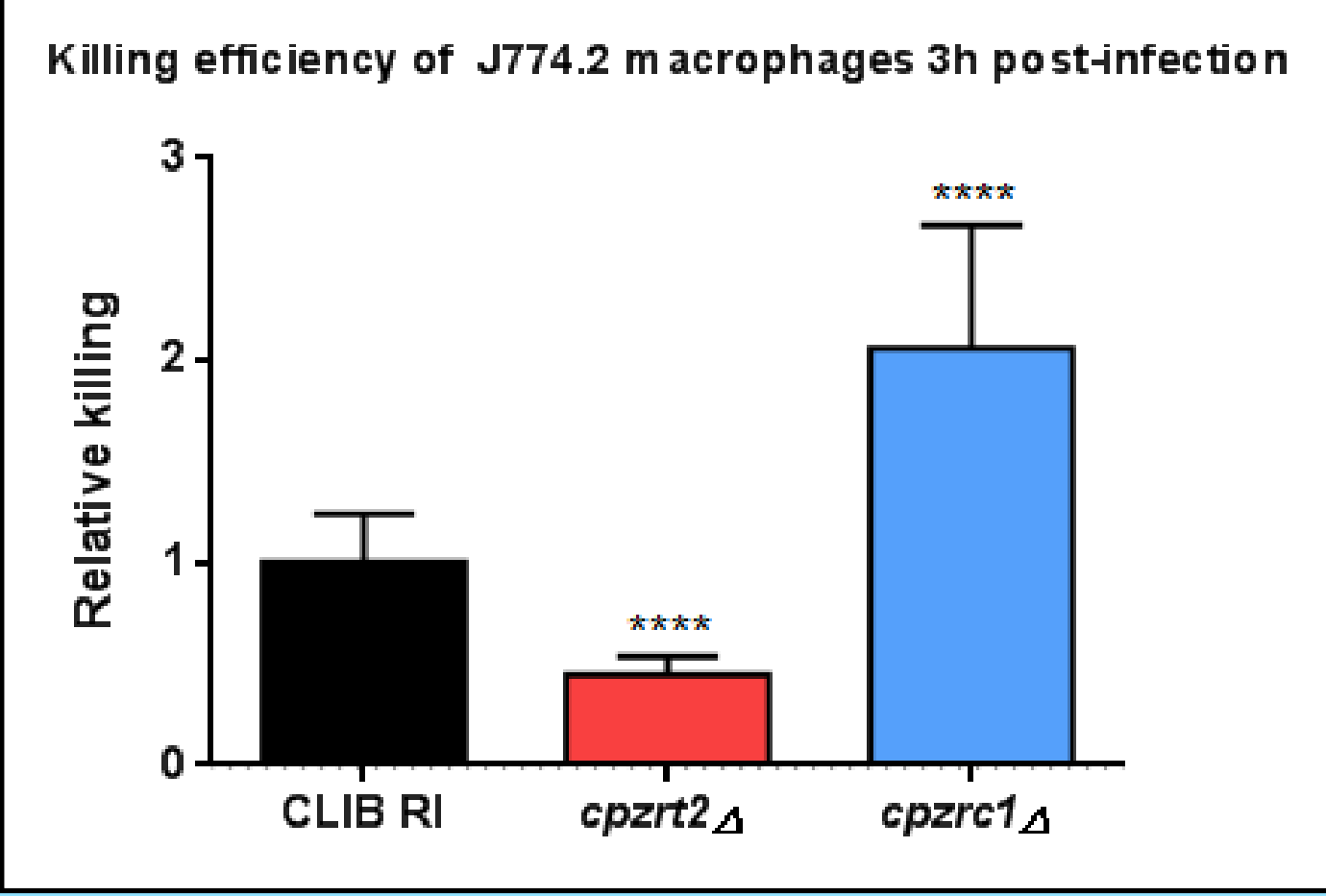

This was in line with our findings regarding fungal elimination by J774.2 cells. Killing of cpzrt2 $\Delta$ was less effective, while in the case of cpzrc1 $1 \Delta$ was more prominent, than the one of the control.

SUMMARY AND HYPOTESIS We found that the lack of the low affinity (fast) zinc transporter resulted in less viability during zinc starvation $(0.05 \mathrm{mM})$ that coupled with higher resistance to uptake and elimination by $\mathbf{J 7 7 4 . 2}$ phagocytes. At the same time the absence of the vacuolar zinc importer decreased the viability of $C$. parapsilosis at high zinc concentration $(5 \mathrm{mM})$ and the deletion strain was less resistant to phagocytosis and killing. This suggests that $\mathbf{J 7 7 4 . 2}$ macrophages might increase the zinc concentration in phagolysosomes to eliminate $C$. parapsilosis To prove our hypothesis, we are planning to apply fluorescent dyes to measure phagolysosomal zinc concentration.

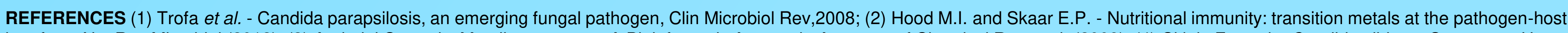

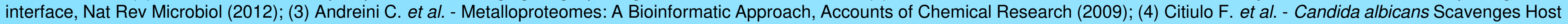

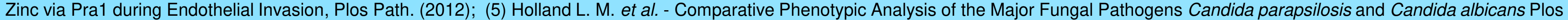
Path. (2014) 